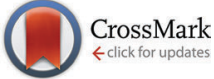

Cite this: Chem. Commun., 2015, 51,4623

Received 14th January 2015, Accepted 11th February 2015

DOI: $10.1039 / \mathrm{c} 5 \mathrm{cc} 00378 d$

www.rsc.org/chemcomm

\section{Structure-tunable Janus fibers fabricated using spinnerets with varying port angles $\dagger$}

\author{
Gaoyun Chen, $\neq^{\mathrm{a}}$ Ying $\mathrm{Xu}, \ddagger^{\mathrm{bc}}$ Deng-Guang Yu, ${ }^{* \mathrm{c}}$ Dao-Fang Zhang, ${ }^{\mathrm{b}}$ \\ Nicholas P. Chatterton ${ }^{a}$ and Kenneth N. White ${ }^{* a}$
}

The preparation of Janus fibers using a new side-by-side electrospinning process is reported. By manipulating the angle between the two ports of the spinneret emitting the working fluids, Janus nanofibers with tunable structures in terms of width, interfacial area and also volume of each side can be easily fabricated.

Among the different "top-down" processes for nanofabrication, electrohydrodynamic atomization processes (EHDA, including electrospinning, electrospraying and e-jetting printing) have the unique capability of simultaneous modulation of sizes, shapes and compartmentalization of their nanoproducts. ${ }^{1,2}$ This ability has been broadly exploited for the generation of core-shell nanostructures, both in the form of electrospun fibers, nanotubes ${ }^{3-5}$ and electrosprayed particles or bubbles. ${ }^{6-8}$ Most recently, even complex structures such as tri-axial nanofibers have also been investigated using multi-fluid electrospinning processes..$^{9-12}$ In sharp contrast, there are still very limited studies about the generation of sideby-side structures using these EHDA processes..$^{13-21}$ To create structures with double compartments, two types of relationships between components are feasible, one is the exterior and interior (i.e. a core-shell structure), and the other is side-by-side. The latter, being a heterojunction structure, can have an advantage for designing novel functional nanomaterials over the core-shell structures because it provides an opportunity for both components to interact with their surroundings. ${ }^{22,23}$

Side-by-side electrospinning involves a complex interplay between fluid dynamics, electrodynamics and rheology, and

\footnotetext{
${ }^{a}$ School of Human Sciences, Faculty of Life Sciences and Computing, London Metropolitan University, 166-220 Holloway Road, London N7 8DB, UK. E-mail: kenneth.white@londonmet.ac.uk

${ }^{b}$ School of Environment and Architecture, University of Shanghai for Science and Technology, Shanghai 200093, China

${ }^{c}$ School of Materials Science \& Engineering, University of Shanghai for Science and Technology, Shanghai 200093, P. R. China. E-mail:ydg017@usst.edu.cn

$\dagger$ Electronic supplementary information (ESI) available: Experimental procedures, characterization methods, and Fig. S1-S4. See DOI: 10.1039/c5cc00378d \$ These authors contributed equally to this work.

$\S$ Present address: School of Biological Sciences, Queen's University of Belfast, BT9 5BN, UK.
}

presents a challenge in controlling the movement in unison of two fluids in a side-by-side manner under an electrical field from spinneret to collector. To our knowledge, Gupta and Wilkes were the first to report the preparation of side-by-side polymer nanofibers, made of poly(vinyl chloride)/segmented polyurethane and poly(vinyl chloride)poly(vinylidiene fluoride) using a spinneret consisting of two parallel Teflon capillaries. ${ }^{13}$ Lin et al. reported the preparation of self-crimping side-by-side nanofibers that spontaneously formed curled helical fibers, composed of polyacrylonitrile and polyurethane, using a homemade silicone microfluidic spinneret, which consisted of three capillary channels with two of them combined in another channel in parallel to form the side-by-side outlet. ${ }^{14}$ Later, several publications describe the preparation of side-by-side nanofibers using spinnerets made from two syringes whose needle tips were confined in a side-byside geometry. ${ }^{15-18}$ In all these studies it was possible to control the outlet of the double fluids in a parallel manner for successful preparation of Janus nanofibers.

Using a different approach, we report here side-by-side electrospinning in which a series of spinnerets with varying port angles were exploited to create structure-tunable Janus fibers. Our initial purpose was to prepare high quality integral Janus nanofibers with little or no separation of the two sides, something that can happen very easily owing to mutual repulsion. However, in the process of optimizing the spinneret design we found unexpectedly that the structures of the Janus nanofibers could be manipulated easily, predominantly by varying the port angle. The variations in Janus structure include (1) the surface or volume of the double sides in their fibers, (2) the width of fibers, and also (3) their interfaces.

Fig. 1 demonstrates the side-by-side electrospinning process used. A diagram of the spinneret and the side-by-side process is shown in Fig. 1a, highlighting the spinneret design, the most creative part of an electrospinning system (which also includes a power supply, syringe pumps and a collector). Fig. $1 \mathrm{~b}$ is a digital picture of the homemade side-by-side spinneret with a port angle of $60^{\circ}$. Fig. 1c shows the spinneret connected with the power supply and syringe pumps (the arrangement of the whole apparatus can be found in ESI $\dagger$ ); highly elastic silicon tubes were exploited to direct the fluids from the syringe to the outlets of the spinneret 


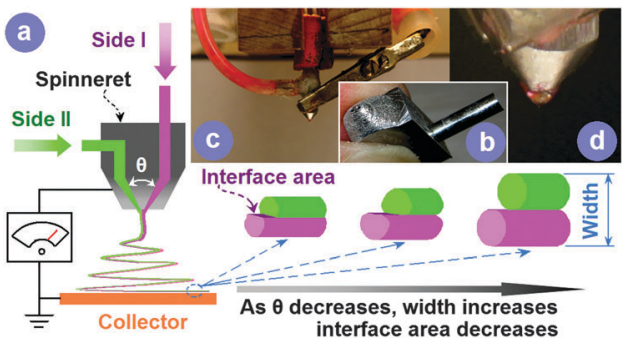

Fig. 1 Implementation of the side-by-side electrospinning process. (a) A diagram of the spinneret and the side-by-side process; (b) a picture of a spinneret with a port angle of $60^{\circ}$; (c) the connection of the spinneret with the fluids and high power supply (the arrangement of the complete apparatus can be found in ESI $\dagger$ ); (d) a digital picture of the compound Taylor cone $\left(\theta=60^{\circ}\right)$.

and an alligator clip was used to connect the spinneret to the power supply for charging the working fluids.

Two electrospinnable working fluids were prepared for implementing the side-by-side electrospinning. One working fluid consisted of $10 \%(\mathrm{w} / \mathrm{v})$ polyvinylpyrrolidone K60 (PVP K60) and $1 \%(\mathrm{w} / \mathrm{v})$ rhodamine $\mathrm{b}\left(\mathrm{C}_{28} \mathrm{H}_{31} \mathrm{~N}_{2} \mathrm{O}_{3} \mathrm{Cl}\right)$ in a mixed solvent of ethanol and $N, N$-dimethylacetamide (DMAc) with a volume ratio of $8: 2$, and the other fluid was composed of $13 \%(\mathrm{w} / \mathrm{v})$ Eudragit ${ }^{\mathbb{R}}$ L100 (EL100, an anionic copolymer based on methacrylic acid and methyl methacrylate) and 2\% (w/v) 8-anilino-1-naphthalenesulfonic acid ammonium salt (ANS-NH4, $\mathrm{C}_{16} \mathrm{H}_{16} \mathrm{~N}_{2} \mathrm{O}_{3} \mathrm{~S}$ ) in a mixture of ethanol and DMAc with a volume ratio of $9: 1$. Under an optimized electrospinning condition (an applied voltage of $12 \mathrm{kV}$, a fixed collection distance of $15 \mathrm{~cm}$ and flow rate of $1.0 \mathrm{~mL} \mathrm{~h}^{-1}$ ), the resultant nanofibers of PVP and EL 100 have an average diameter of $0.72 \pm 0.17 \mu \mathrm{m}$ and $0.83 \pm 0.12 \mu \mathrm{m}$, respectively, when electrospun individually (ESI $\dagger$ ). Using similar conditions for side-by-side electrospinning, with an equal flow rate of $1.0 \mathrm{~mL} \mathrm{~h}^{-1}$ for both side fluids, a stable compound Janus Taylor cone could be observed, as shown in Fig. 1d, with one red side of PVP K60/rhodamine b and the other side of EL100/ANS-NH4.

A typical FESEM image of a Janus nanofiber mat prepared using the spinneret with a port angle of $60^{\circ}$ is shown in Fig. 2a. Three points (A, B and C) were selected to conduct element composition analysis using an energy dispersive spectrometer (EDS), the results of which are given in Fig. 2b-d sequentially. Although a very small amount of 0.6 (wt\%) sulphur was detected in the analysis shown in Fig. 2b, its presence demonstrates that point A is in the side of EL100/ANS-NH4. Similarly, the existence of chlorine in the spectrum shown in Fig. 2c suggested that point $\mathrm{B}$ is in the side of PVP K60/rhodamine b. As anticipated, the spectrum of point $\mathrm{C}$ in Fig. $2 \mathrm{~d}$ shows the co-existence of sulphur and chlorine because it is a result from the whole Janus nanofiber in a lateral topology.

Four spinnerets with port angles of $40^{\circ}, 50^{\circ}, 60^{\circ}$ and $70^{\circ}$ were exploited to prepare the Janus nanofibers under the same electrospinning conditions (an applied voltage of $12 \mathrm{kV}$, a fixed collected distance of $15 \mathrm{~cm}$, and a flow rate of $1.0 \mathrm{~mL} \mathrm{~h}^{-1}$ for both fluids). The TEM images of the resultant Janus nanofibers are shown in Fig. 3. As the port angle increased successively from $40^{\circ}$ to $70^{\circ}$, the interface of the two sides became increasingly vaguer.

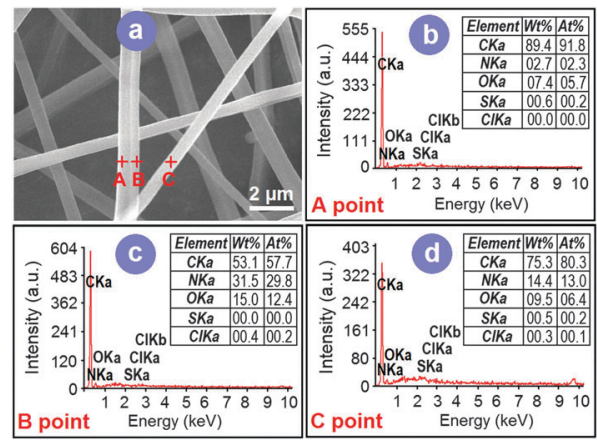

Fig. 2 Demonstration of the Janus structure using EDS. (a) An FESEM image of the Janus nanofibers prepared using a spinneret with a port angle of $60^{\circ}$; (b)-(d) are EDS spectra of the elements contained at points A, B and $C$ of (a) sequentially.

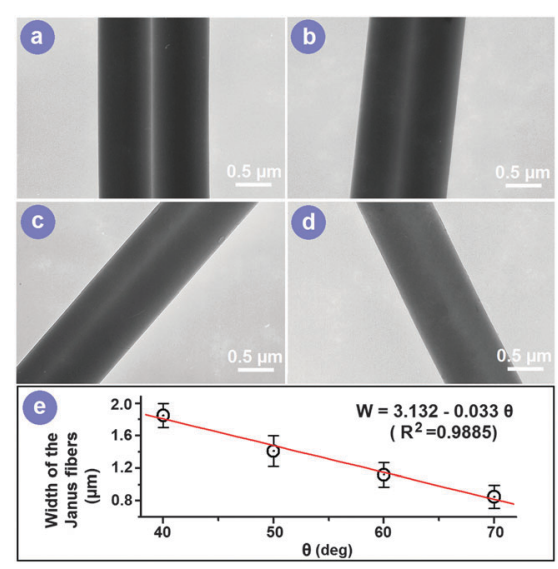

Fig. 3 Tunable width and interfacial area of the Janus nanofibers with change of port angle. (a)-(d) Are TEM images of Janus nanofibers fabricated using spinnerets with a port angle of $40^{\circ}, 50^{\circ}, 60^{\circ}$ and $70^{\circ}$ sequentially. (e) The linear relationship between the width of the Janus nanofiber $(W)$ and the port angle $(\theta)$; the FESEM images for measuring the width of Janus nanofibers can be found in the ESI. $\dagger$

This is reflected by the interfacial central line between the two components which became increasingly wider and diffuse, as shown in Fig. 3a through b, c to d, while the fiber width became narrower. Data from FESEM images indicated that they have widths of $1.81 \pm 0.15 \mu \mathrm{m}, 1.42 \pm 0.19 \mu \mathrm{m}, 1.12 \pm 0.15 \mu \mathrm{m}$ and $0.87 \pm 0.13 \mu \mathrm{m}$ respectively (ESI $\dagger$ ). A linear relationship between the width of the Janus nanofiber $(W)$ and the port angle $(\theta)$ was evident, $W=3.312-0.033 \theta\left(R^{2}=0.9885\right)$ (Fig. 3e).

In the side-by-side electrospinning process, the two fluid jets emanate from their own ducts, carrying the same electrostatic charge. It may be expected that the mutual electrostatic repulsion of the jets would keep the two solutions from mixing, ${ }^{13,14}$ or that the mutual electrostatic repulsion may also result in separation of the two sides before they are solidified, resulting in failure to form Janus nanofibers with integrated structures. However, we have shown that having an arrangement of the ports at an angle to induce the two fluids to converge at their outlet of the spinneret's nozzle facilitates the formation of integrated Janus nanofibers. A bigger port angle would result in a relatively larger diffusion 


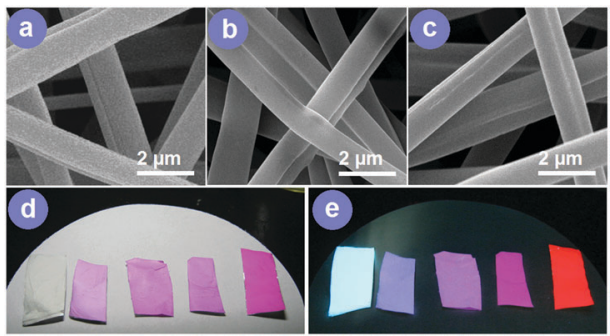

Fig. 4 Tunable volume of both sides in the Janus nanofibers using different fluid flow rate ratios. SEM images of Janus fibers produced with a spinneret with port angle $50^{\circ}$ and with flow rate ratio of the EL100/ANS-NH4 side to the PVP/rhodamine $b$ side of $0.5: 1.5,1: 1$, and $1.5: 0.5$ for (a)-(c) respectively. Digital images of nanofiber mats viewed under natural light (d) and ultraviolet light of $\lambda=364 \mathrm{~nm}$ (e); mats from left to right are nanofibers of EL100/ANS-NH4, Janus nanofibers as prepared in (a) to (c), and PVP/rhodamine nanofibers.

of the miscible fluids, and in turn the possible formation of a region between the two sides with gradient distributions of the compositions. But because the two jets would still move to the collector in a parallel manner after they are ejected from the Taylor cone, the mixing or diffusion would be limited. ${ }^{13,19}$

In practice, the structure of Janus nanofibers can be tuned in a controlled manner appropriate to their potential application, through manipulation of the port angles of the spinneret. For example, when the Janus nanofibers are intended to be used as materials for solar cell systems, in which the existence of a sharp interface between the phases is crucial, ${ }^{20}$ they can be fabricated using a spinneret with a small port angle to create a structure such as those in Fig. 3a. But when the nanofibers are designed to achieve multiple functional performances with one side to provide strong mechanical properties and the other side to furnish another property, a structure like those shown in Fig. 3d, prepared using a spinneret with a large port angle, should be adopted.

For some potential applications of Janus structures, often each part is to have its own function but together they act as a whole to provide a combined action. For example, the controlled release of multiple drugs for a combined therapy. In these cases, being able to tune the volume of both sides permits the easily tailoring of the Janus fibers' functional performance. Using the same working fluids mentioned above and also the same experimental conditions but different flow rate ratios, three variants of Janus nanofibers were fabricated (Fig. 4a-c). Although they showed a similar pink color under natural light (Fig. 4d), they had varying colors under an ultraviolet light with a wavelength of $364 \mathrm{~nm}$, from light blue to pink and to amaranth when the flow rate ratio of the EL100/ANS-NH4 side to $\mathrm{PVP} /$ rhodamine side changed from $0.5: 1.5$ to $1: 1$, and 1.5: 0.5 in sequence (Fig. 4e). The monolithic nanofibers prepared from single working fluids of either side showed the distinct color of the encapsulated single dye under ultraviolet light in Fig. 4e. As expected, the two dyes existed in their polymer matrices in an amorphous state (ESI $\dagger$ ), suggesting that the side-by-side electrospinning processes described here can be exploited for the preparation of Janus structural composites, which are popular for designing new functional materials. ${ }^{23}$
Particularly, the amorphous status is highly desirable for improving the dissolution of poorly water soluble drugs (one of the most difficult challenges in pharmaceutics), ${ }^{24-26}$ and thus the present processes should provide a new platform for developing drug delivery systems for combined therapy from poorly water soluble active pharmaceutical ingredients. Moreover, these tunable Janus fiber structures should have good potential applications for fibrous guided tissue regeneration membranes because not only multiple functional ingredients such as anti-inflammatory agents, adhesive components for cell attachment and even nutritional ingredients can be loaded in them, but also one side can be managed to degrade to make way for proliferating cells. ${ }^{27}$

No matter how complex the electrospinning process is, it is totally a physical process. However, few studies have reported on the process of electrospinning from an engineering standpoint. The mass transfer, energy transfer and also momentum transfer during electrospinning are still waiting to be characterized. The present study provides a good methodology for investigating the momentum transfer when the electrospinning process is initiated.

In summary, we demonstrated that the port angle of the spinneret has a significant influence on the character of the resultant Janus nanofibers. By manipulating this parameter, Janus nanofibers with tunable structures in terms of width, interfacial area and also volume of each side can be fabricated. These tunable Janus structures should provide a new platform for designing new functional materials and developing new nanostructures with multiple functionalities. We are further exploiting this approach to fabricate Janus structures from two immiscible working solutions, or with only one of the two fluids electrospinnable.

This work was supported at London Metropolitan University by the Emerald Fund, and by the National Science Foundation of China (No. 51373101), the Natural Science Foundation of Shanghai (No. 13ZR1428900) and the Key Project of the Shanghai Municipal Education Commission (No. 13ZZ113).

\section{Notes and references}

1 K. J. Lee, J. Yoon and J. Lahann, Curr. Opin. Colloid Interface Sci., 2011, 16, 195.

2 M. C. George and P. V. Braun, Angew. Chem., Int. Ed., 2009, 48, 8606.

3 J. T. McCann, D. Li and Y. Xia, J. Mater. Chem., 2005, 15, 735.

4 D. G. Yu, L. M. Zhu, C. Branford-White, S. W. A. Bligh and K. White, Chem. Commun., 2011, 47, 1216.

5 D. G. Yu, X. Wang, X. Y. Li, W. Chian, Y. Li and Y. Z. Liao, Acta Biomater., 2013, 9, 5665.

6 R. G. Chaudhuri and S. Paria, Chem. Rev., 2012, 112, 2373.

7 I. G. Loscertales, A. Barrero, I. Guerrero, R. Cortijo, M. Marquez and A. M. Canan-Calvo, Science, 2002, 295, 1695.

8 Z. Ekemen, Z. Ahmad, M. Edirisinghe and E. Stride, Macromol. Mater. Eng., 2011, 296, 8.

9 S. Bhaskar and J. Lahann, J. Am. Chem. Soc., 2009, 131, 6650.

10 S. Jiang, G. Duan, E. Zussman, A. Greiner and S. Agarwal, ACS Appl. Mater. Interfaces, 2014, 6, 5918.

11 J. D. Starr and J. S. Andrew, J. Mater. Chem. C, 2013, 1, 2529.

12 H. Chen, Y. Zhao, Y. Song and L. Jiang, J. Am. Chem. Soc., 2008, 130, 7800 .

13 P. Gupta and G. L. Wilkes, Polymer, 2003, 44, 6353.

14 T. Lin, H. Wang and X. Wang, Adv. Mater., 2005, 17, 2699. 
15 S. Chen, H. Hou, P. Hu, J. H. Wendorff, A. Greiner and S. Agarwal, Macromol. Mater. Eng., 2009, 294, 781.

16 Z. Liu, D. D. Sun, P. Guo and J. O. Leckie, Nano Lett., 2007, 7, 1081. 17 J. D. Starr, M. A. K. Budi and J. S. Andrew, J. Am. Ceram. Soc., 2015, 98, 12. 18 J. D. Starr and J. S. Andrew, Chem. Commun., 2013, 49, 4151.

19 T. M. Squires and S. R. Quake, Rev. Mod. Phys., 2005, 77, 977.

20 Z. Nie, W. Li, M. Seo, S. Xu and E. Kumacheva, J. Am. Chem. Soc., 2006, 128, 9408.

21 X. Y. Ji, Q. Zhang, F. X. Liang, Q. N. Chen, X. Z. Qu, C. L. Zhang, Q. Wang, J. L. Li, X. M. Song and Z. Z. Yang, Chem. Commun., 2014, 50, 5706.
22 L. L. Zhao, L. J. Zhu, Y. Chen, Q. Wang, J. L. Li, C. L. Zhang, F. X. Liang, X. Z. Qu and Z. Z. Yang, Chem. Commun., 2013, 49, 6161. 23 F. X. Liang, C. L. Zhang and Z. Z. Yang, Adv. Mater., 2014, 26, 6944. 24 F. L. Lopez, G. C. Shearman, S. Gaisford and G. R. Williams, Mol. Pharmaceutics, 2014, 11, 4327.

25 J. Yan, Y. H. Wu, D. G. Yu, G. R. Williams, S. M. Huang, W. Tao and J. Y. Sun, $R S C A d v$., 2014, 4, 58265.

26 D. G. Yu, G. R. Williams, X. Wang, X. K. Liu, H. L. Li and S. W. A. Bligh, RSC Adv., 2013, 3, 4652.

27 T. Dvir, B. P. Timko, D. S. Kohane and R. Langer, Nat. Nanotechnol., 2011, 6, 13. 